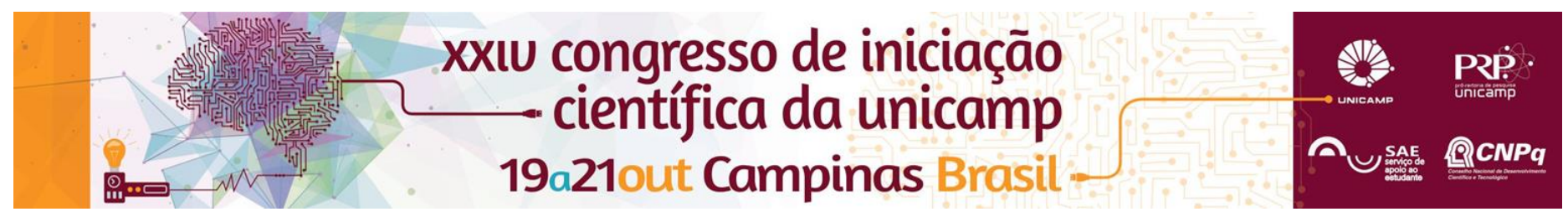

\title{
MELANOMA ORAL: RELATO DE DOIS CASOS
}

\author{
Gabriel Brandão Alexandre*, Homero Rodrigues Passos, Suzana Shinomia, Maria Letícia Cintra, Albina Altemani, \\ Fernanda V. Mariano.
}

\section{Resumo}

O melanoma bucal é um tumor potencialmente agressivo de origem melanocítica, que surge através de uma lesão melanocítica benigna ou de melanócitos da mucosa. Apresentamos dois casos de pacientes com melanoma oral e objetivamos chamar atenção para esta neoplasia neste sítio anatômico, visto comportamento agressivo que apresenta.

\section{Palavras chave: \\ Melanoma oral, características clínicopatológicas, agressividade}

\section{Introdução}

O Melanoma é considerado uma neoplasia maligna composta por melanócitos, que são células derivadas da crista neural e que produzem o pigmento melanina. Apesar da maioria dos melanomas surgirem na pele, eles podem também ocorrer em superfícies mucosas como as das cavidades oral e nasal, além de outras regiões. Somente cerca de $55 \%$ ocorrem na região de cabeça e pescoço e destes, $1,3 \%$ dos melanomas acometem as mucosas. O restante atinge $18 \%$ no trato genital feminino, $23,8 \%$ na região anal/retal e $2,8 \%$ no trato urinário.

Em relação à etiopatogenia do $\mathrm{MC}$, acredita-se que ela esteja associada a diversos fatores predisponentes, como por exemplo, exposição à luz solar, pele clara, lesões melanocíticas benignas, história de câncer de pele e baixa imunidade. Já quanto ao $\mathrm{MO}$, poucos fatores etiológicos foram propostos, sendo que aparentemente esta neoplasia parece surgir de uma lesão melanocítica benigna ou de melanócitos normais da mucosa. Em relação ao MS, poucas causas foram identificadas, porém acredita-se que esta neoplasia inicia-se provavelmente de novo ou de uma lesão pré-existente. Além disso, uma possível associação entre MS e carcinógenos inalados já foi sugerida na literatura.

\section{Resultados}

RELATO: Apresentamos dois casos de pacientes que apresentaram melanoma oral. O primeiro paciente, gênero masculino, tinha 64 anos e exibia lesão em gengiva superior esquerda posterior medindo aproximadamente 2 $\mathrm{cm}$. A segunda paciente, gênero feminino, apresentava lesão em gengiva superior antero-lateral. Ambos desconheciam tempo de evolução da lesão. O primeiro paciente foi tratado por cirurgia, mas depois de 1 ano de evolução foi a óbito pela doença. A segunda paciente não aderiu ao tratamento e manteve-se perdida de vista.

\section{Conclusões}

Os presentes casos relatam a importância de se ilustrar neoplasias incomuns da cavidade oral, mas que despertam a atenção do clínico devido à agressividade.

\section{Agradecimentos}

Os autores agradecem a Ana Cláudia Sparapani Piazza, Arethusa Souza, Fabiana Cassarotti e Luzia Magalhães Alves pela assistência técnica.

\section{Este estudo foi suportado por:}

FAPESP: 2011/23204-5, FAPESP: 2011/23366-5

FAPESP: 2016/07753-2

Coordenação de Aperfeiçoamento Pessoal e de Nível Superior (CAPES-PNPD).

\section{Conflito de interesse}

Os autores declaram que não há conflito de interesse.

1. Hicks MJ, Flaitz CM. Oral mucosal melanoma: epidemiology and pathobiology. Oral Oncol. 2000;36:152-169.

2. Wada H, Nemoto K, Ogawa Y, et al. A multi-institutional retrospective analysis of external radiotherapy for mucosal melanoma of the head and neck in Northern Japan. Int J Radiat Oncol Biol Phys. 2004;59:495-500.

3. Chang AE, Karnell LH, Menck HR. The National Cancer Data Base report on cutaneous and noncutaneous melanoma: a summary of 84,836 cases from the past decade. The American College of Surgeons Commission on Cancer and the American Cancer Society. Cancer. 1998;83:1664-1678.

4. Vikey AK, Vikey D. Primary malignant melanoma of head and neck: a comprehensive review of literature. Oral Oncol. 2012;48: 399-403.

5. Sortino-Rachou AM, Cancela Mde C, Voti L, Curado MP. Primary oral melanoma: population-based incidence. Oral Oncol. 2009;45:254-258.

6. Batsakis JG, Regezi JA, Solomon AR, Rice DH. The pathology of head and neck tumors: mucosal melanomas. Head Neck Surg. 1982;4:404-418.

7. Barker BF, Carpenter WM, Daniels TE, et al. Oral mucosal melanomas: the WESTOP Banff workshop proceedings. Western Society of Teachers of Oral Pathology. Oral Surg Oral Med Oral Pathol Oral Radiol Endod. 1997;83:672679

8. Nandapalan V, Roland NJ, Helliwell TR, Williams EMI, Hamilton JW, Jones AS. Mucosal melanoma of the head and neck. Clin Otolaryngol. 1998;23:107116

9. Patrick RJ, Fenske NA, Messina JL. Primary mucosal melanoma. J Am Acad Dermatol. 2007;56:828-834

10. Rapini RP, Golitz LE, Greer RO Jr, Krekorian EA, Poulson T. Primary malignant melanoma of the oral cavity. A review of 177 cases. Cancer. 1985;55:15431551 .

11. Patel SG, Prasad ML, Esrig M, Singh B, Shaha AR, Kraus DH. Primary mucosal malignant melanoma of the head and neck. Head Neck. 2002;24:247257.

12. Boulaadas M, Benazzou S, Mourtada F, Sefiani S, Nazih N, Essakalli L, Kzadri M. Primary oral malignant melanoma. J CraniofacSurg 2007; 18: 1059 61. 